\title{
Chemical Management of Whiteflies in Sunflower
}

\author{
Praveen B. Huded, V.N. Ghante* and Vikas Kulkarni \\ Main Agricultural Research Station, UAS, Raichur, Karnataka, India \\ *Corresponding author
}

\section{A B S T R A C T}

\begin{tabular}{|l|}
\hline K e y w o r d s \\
Whiteflies, \\
Sunflower, \\
Insecticides, \\
Diafenthiuron
\end{tabular}

\section{Introduction}

Sunflower (Helianthus annuus L.) popularly known as "Surajmukhi" as it follows the movement of the sun. It belongs to the genus 'Helianthus', family 'Asteraceae' (Compositae). The name has its origin in Greek "Helios" means "Sun" and "Anthois" means "flower". It is native to southern parts of USA and Mexico (Heiser, 1951). Sunflower is an important oilseed crop in India. Sunflower originated in Southern United States and Mexico from where it was introduced into Europe and later into former Union of Soviet Socialist Republics (USSR). Majority of the present day varieties are grown all over the world. The crop originated from former USSR. In India, sunflower crop was introduced in 1969.

In India, sunflower is being grown over an area of $0.52 \mathrm{~m}$ ha with a production of 0.34 million tonnes and the productivity of $643 \mathrm{~kg}$ per ha. In Karnataka sunflower is being grown over an area of $0.35 \mathrm{~m}$ ha with a production of 0.20 million tonnes and the productivity of $579 \mathrm{~kg}$ per ha (Anon., 2016).

Sunflower is one of the important edible oilseed crop grown in the world after soybean and groundnut. It is an important source of edible and nutritious oil. Almost every part of sunflower has commercial value. It is used in the manufacturing paints, resins, plastics, soap 
and cosmetics. Oil cake is rich in high quality protein ( $40-44$ per cent) and is used as cattle and poultry feed. It is affected by several biotic and abiotic stresses.

Among several biotic stresses for successful sunflower production, susceptibility to insect pests and diseases are major constraints. The crop is attacked by a number of insect-pests and as many as 251 insect species are known to attack this crop the world over (Rajmohan,1976). In India, major insect-pests associated with this crop are capitulum borer Helicoverpa armigera (Hübner), green semilooper Thysanoplusia orichalcea Fab., Bihar hairy caterpillar Spilosoma obliqua (Walker), tobacco caterpillar Spodoptera litura (Fab.), cutworm Agrotis sp. and green leaf hopper Amrasca biguttula biguttula (Basappa,1995).

The loss in seed yield due to these pests is estimated to be around 24.9 per cent (Anonymous, 2013). Sucking pests like whiteflies, leafhoppers and thrips contribute to considerable extent of loss to the crop. Sucking pests generally occurs at early stage of crop and its damage continues till grain filling stage. Among all sucking pests, leaf hopper causes major loss of 46 per cent (Anon., 1997), thrips are involved in transmission of sunflower bud necrosis virus (Chander Rao, 2002) and whiteflies are of prime importance which under favourable conditions pose threat to the crop, which do not cause direct damage but cause enormous loss indirectly as vector of viral disease (Govindappa et al., 2011).

Recently, whitefly has emerged as the new potential sucking insect pest of sunflower and also acting as the vector of leaf curl begomovirus in Northern Karnataka, India. This has attracted lot of attention of the entomologists and pathologists, as it affects the productivity of sunflower an important oilseed crop in the country (Katti, 2007). Sunflower leaf curl disease transmitted by whitefly was noticed for the first time in the country and the disease was recorded on sunflower hybrid 'Sun breed - 275' up to 40 per cent disease incidence in the fields of Main Agricultural Research Station (MARS), University of Agricultural Sciences, Raichur, Northern Karnataka, during Rabi season of 2009 (Govindappa et al., 2011).

The whitefly infestation has been noticed in an endemic form consecutively for the last three years in sunflower growing areas of Northern districts of Karnataka. The disease severity ranged from 10 - 58 per cent and correspondingly the increased population of $B$. tabaci (up to 200 per leaf) was observed with an average of $14.7 \mathrm{~B}$. tabaci per leaf both in open pollinated varieties and hybrids. Further, the incidence of SuLCV was more in Koppal $(11.16 \%)$ district fallowed by Raichur (7.41 $\%)$ and Ballari (6.34 \%) district (Vindhyashree, 2014).

Farmers are unable to control the whiteflies even with the repeated insecticidal sprays with conventional insecticides which resulted in pollution of natural resources and also development of resistance. Hence, there is need for evaluating the newer insecticides that may be effective against whitefly in sunflower.

\section{Materials and Methods}

The field experiment was laid in randomized block design during 2017 comprising nine treatments and three replications. Nine different chemicals namely Azadirachtin $0.03 \%$ at $5 \mathrm{ml} / 1$, Buprofezin $25 \% \mathrm{SC}$ at $1 \mathrm{ml} / 1$, Diafenthiuron $50 \% \mathrm{WP}$ at $1 \mathrm{~g} / \mathrm{l}$, Chlothanadin $50 \%$ WDG at $0.1 \mathrm{~g} / \mathrm{l}$, Fipronil $5 \% \mathrm{SC}$ at $1 \mathrm{ml} / 1$, Flonicamide $50 \% \mathrm{WG}$ at $0.4 \mathrm{~g} / \mathrm{l}$, Profenophos $50 \% \mathrm{EC}$ at $2 \mathrm{ml} / 1$, Triazophos $40 \%$ EC at $2 \mathrm{ml} / 1$ were evaluated against whitefly on sunflower 
hybrid KBSH 44 (Table 1 and 2). Two sprays were taken up at 50 DAS and 70 DAS. Observations on whitefly were made on six leaves comprising of two leaves each from top, middle and bottom portion of five randomly selected plants from each plot. Population of whiteflies was recorded at one day before treatment and one, three, five, seven and ten days after treatment. The mean population of whitefly was worked out and subjected to statistical analysis.

\section{Results and Discussion}

Among various insecticides evaluated, diafenthiuron 50 \% WP @ 1 g/l recorded significantly least whitefly population $(12.00$ and 10.43 whitefly per plant after first and second spray respectively) followed by triazophos $40 \%$ EC @ 2 ml/l (12.48 and 11.22 whitefly per plant after first and second spray respectively).

The next best treatment was flonicamid 50\% WG @ $0.4 \mathrm{~g} / \mathrm{l}$ (16.25 and 13.80 whitefly per plant after first and second spray respectively) which was on par fipronil 5\% SC @ $1 \mathrm{ml} / \mathrm{l}$ (16.57 and 13.77 whitefly per plant after first and second spray respectively), which was followed by buprofezin 25\% SC @ $1 \mathrm{ml} / \mathrm{l}$ (17.05 and 14.46 whitefly per plant after first and second spray respectively). The chemicals proved to be effective up to seven days after spraying.

Diafenthiuron $50 \%$ WP having mean per cent reduction over untreated control of whitefly was 57.00 and 60.70 per cent after first and second sprays respectively followed by triazophos $40 \% \mathrm{EC}$ which had mean per cent reduction over untreated control of whitefly with 55.28 and 57.72 per cent after first and second spray respectively.
These treatments were followed by clothianidin $50 \%$ WG, profenophos $50 \%$ EC and azadirachtin $0.03 \%$. Untreated control recorded highest pest population. The present findings were in agreement with Kharel et al., (2016) who reported that diafenthiuron $50 \mathrm{WP}$ @ $312 \mathrm{~g}$ a.i./ha was most promising treatment in reducing population of whitefly.

Similarly, Dhaka (2016) reported that the treatment with spiromesifen 240 SC @ 150 ml.a.i./ha and diafenthiuron 50 WP @ $312.5 \mathrm{~g}$ a.i./ha were found very effective against sucking pests. Gopal Das and Tarikul Islam (2014) reported that fipronil and buprofezin proved to be the superior against jassids and whiteflies. Further, Sanjeev Kumar and Gill (2010) reported that triazophos 40 EC @ 1.0 1/ha recorded lowest population of whiteflies with 3.67/plant as against 10.99/plant in control.

Similarly, Kumar et al., (2001) reported that triazophos $0.05 \%$ was found superior in controlling the whitefly population in brinjal and resulted in 75.22 per cent reduction in population over control. Seed yield significantly higher seed yield of 1346 and $1301 \mathrm{~kg} / \mathrm{ha}$ was recorded in diafenthiuron 50 \%WP @ $1 \mathrm{~g} / 1$ and triazophos $40 \% \mathrm{EC} @ 2$ $\mathrm{ml} / \mathrm{l}$ respectively.

The next best treatment was flonicamid 50\% WG@0.4 g/l (1176 kg/ha)which was on par with and buprofezin 25\% SC @ 1 ml/l (1173 $\mathrm{kg} / \mathrm{ha}$ ) and fipronil 5\% SC @ $1 \mathrm{ml} / \mathrm{l}$ (1169 $\mathrm{kg} / \mathrm{ha}$ ). These were followed by profenophos $50 \%$ EC @ 2 ml $/ 1$, clothianidin 50\% WG @ $0.1 \mathrm{~g} / \mathrm{l}$ and azadirachtin $0.03 \%$ @ $\mathrm{ml} / \mathrm{l}$ which recorded 1136, 1130 and $1125 \mathrm{~kg} / \mathrm{ha}$ respectively. The untreated control recorded lower yield $(852 \mathrm{~kg} / \mathrm{ha})$. 
Table.1 Evaluation of chemical insecticides for the management of whiteflies in sunflower (First spray)

\begin{tabular}{|c|c|c|c|c|c|c|c|c|c|c|}
\hline \multirow{2}{*}{$\begin{array}{l}\text { Sl. } \\
\text { No. }\end{array}$} & \multirow[t]{2}{*}{ Treatment } & \multirow{2}{*}{$\begin{array}{l}\text { Dosage } \\
(\mathrm{ml} \text { or } \mathrm{g} / \mathrm{l})\end{array}$} & \multicolumn{6}{|c|}{ Whitefly population* (per six leaves) } & \multirow[t]{2}{*}{ Mean } & \multirow{2}{*}{$\begin{array}{l}\text { Per cent } \\
\text { reduction } \\
\text { over control }\end{array}$} \\
\hline & & & 1 DBS & 1 DAS & 3 DAS & 5 DAS & 7 DAS & 10 DAS & & \\
\hline 1. & Triazophos $40 \% \mathrm{EC}$ & $2 \mathrm{ml}$ & $\begin{array}{l}29.21 \\
(5.45)\end{array}$ & $\begin{array}{c}18.10 \\
(4.34) \mathrm{a}\end{array}$ & $\begin{array}{c}11.21 \\
(3.42) \mathrm{a}\end{array}$ & $\begin{array}{c}6.74 \\
(2.69) \mathrm{b}\end{array}$ & $\begin{array}{c}4.26 \\
(2.18) \mathrm{a}\end{array}$ & $\begin{array}{c}5.34 \\
(2.42) \mathrm{b}\end{array}$ & 12.48 & 55.28 \\
\hline 2. & $\begin{array}{c}\text { Profenophos } 50 \% \\
\text { EC }\end{array}$ & $2 \mathrm{ml}$ & $\begin{array}{l}28.96 \\
(5.43)\end{array}$ & $\begin{array}{c}23.57 \\
(4.91) c\end{array}$ & $\begin{array}{c}17.60 \\
(4.25) \mathrm{c}\end{array}$ & $\begin{array}{c}13.54 \\
(3.75) \mathrm{d}\end{array}$ & $\begin{array}{c}9.83 \\
(3.21) \mathrm{cd}\end{array}$ & $\begin{array}{c}10.91 \\
(3.38) \mathrm{e}\end{array}$ & 17.40 & 37.65 \\
\hline 3. & Fipronil $5 \% \mathrm{SC}$ & $1 \mathrm{ml}$ & $\begin{array}{l}30.21 \\
(5.54)\end{array}$ & $\begin{array}{c}22.32 \\
(4.78) b\end{array}$ & $\begin{array}{c}16.35 \\
(4.10) b c\end{array}$ & $\begin{array}{c}12.29 \\
(3.58) \mathrm{cd}\end{array}$ & $\begin{array}{c}8.58 \\
(3.01) \mathrm{bc}\end{array}$ & $\begin{array}{c}9.66 \\
(3.19) \mathrm{c}\end{array}$ & 16.57 & 40.63 \\
\hline 4. & Buprofezin $25 \% \mathrm{SC}$ & $1 \mathrm{ml}$ & $\begin{array}{l}30.45 \\
(5.56)\end{array}$ & $\begin{array}{c}22.87 \\
(4.83) \mathrm{bc}\end{array}$ & $\begin{array}{c}16.81 \\
(4.16) b c\end{array}$ & $\begin{array}{c}12.84 \\
(3.65) \mathrm{cd}\end{array}$ & $\begin{array}{c}9.13 \\
(3.10) \mathrm{bc}\end{array}$ & $\begin{array}{c}10.21 \\
(3.27) \mathrm{d}\end{array}$ & 17.05 & 38.91 \\
\hline 5. & Azadirachtin $0.03 \%$ & $5 \mathrm{ml}$ & $\begin{array}{l}30.45 \\
(5.56)\end{array}$ & $\begin{array}{l}24.85 \\
(5.03) \mathrm{d}\end{array}$ & $\begin{array}{l}18.85 \\
(4.40) \mathrm{cd}\end{array}$ & $\begin{array}{c}14.84 \\
(3.92) \mathrm{e}\end{array}$ & $\begin{array}{l}11.13 \\
(3.41) \mathrm{d}\end{array}$ & $\begin{array}{l}12.21 \\
(3.57) \mathrm{f}\end{array}$ & 18.72 & 32.92 \\
\hline 6. & $\begin{array}{c}\text { Flonic amid } 50 \% \\
\text { WG }\end{array}$ & $0.4 \mathrm{~g}$ & $\begin{array}{l}29.62 \\
(5.49)\end{array}$ & $\begin{array}{c}22.07 \\
(4.75) b\end{array}$ & $\begin{array}{c}16.04 \\
(4.07) b\end{array}$ & $\begin{array}{c}12.04 \\
(3.54) \mathrm{c}\end{array}$ & $\begin{array}{c}8.33 \\
(2.97) \mathrm{b}\end{array}$ & $\begin{array}{c}9.41 \\
(3.15) \mathrm{c}\end{array}$ & 16.25 & 41.77 \\
\hline 7. & $\begin{array}{c}\text { Diafenthiuron } 50 \% \\
\text { WP }\end{array}$ & $1 \mathrm{~g}$ & $\begin{array}{l}30.08 \\
(5.53)\end{array}$ & $\begin{array}{c}17.44 \\
(4.24) \mathrm{a}\end{array}$ & $\begin{array}{c}10.13 \\
(3.26) \mathrm{a}\end{array}$ & $\begin{array}{c}5.79 \\
(2.51) \mathrm{a}\end{array}$ & $\begin{array}{c}3.73 \\
(2.06) \mathrm{a}\end{array}$ & $\begin{array}{c}4.81 \\
(2.30) \mathrm{a}\end{array}$ & 12.00 & 57.00 \\
\hline 8. & $\begin{array}{l}\text { Clothianidin } 50 \% \\
\text { WG }\end{array}$ & $0.1 \mathrm{~g}$ & $\begin{array}{l}30.77 \\
(5.59)\end{array}$ & $\begin{array}{c}22.89 \\
(4.84) b c\end{array}$ & $\begin{array}{c}16.91 \\
(4.17) \mathrm{bc}\end{array}$ & $\begin{array}{c}12.46 \\
(3.60) \mathrm{cd}\end{array}$ & $\begin{array}{c}9.56 \\
(3.17) \mathrm{bc}\end{array}$ & $\begin{array}{c}10.64 \\
(3.34) \mathrm{de}\end{array}$ & 17.21 & 38.33 \\
\hline 9. & Control & - & $\begin{array}{l}29.11 \\
(5.44)\end{array}$ & $\begin{array}{l}28.74 \\
(5.41) \mathrm{e}\end{array}$ & $\begin{array}{c}29.27 \\
(5.46) \mathrm{e}\end{array}$ & $\begin{array}{c}26.41 \\
(5.19) \mathrm{f}\end{array}$ & $\begin{array}{c}26.79 \\
(5.22) \mathrm{e}\end{array}$ & $\begin{array}{c}27.11 \\
(5.25) \mathrm{g}\end{array}$ & 27.91 & 0 \\
\hline \multicolumn{3}{|c|}{$\mathrm{SEM} \pm$} & 0.83 & 0.04 & 0.06 & 0.05 & 0.08 & 0.03 & - & - \\
\hline \multicolumn{3}{|c|}{ CD@5\% } & NS & 0.10 & 0.16 & 0.17 & 0.22 & 0.07 & - & - \\
\hline \multicolumn{3}{|c|}{$\mathrm{CV} \%$} & 14.63 & 12.48 & 9.63 & 11.21 & 10.74 & 9.57 & - & - \\
\hline
\end{tabular}

DBS- days before spraying, DAS-days after spraying, Figures in the parenthesis are $\sqrt{x}+0.5$ transformed values, NS= Non significant, Means followed by same letter in the column do not differ significantly by DMRT $(\mathrm{P}=0.05)$, *Means of three replications. 
Table.2 Evaluation of chemical insecticides for the management of whiteflies in sunflower (Second spray)

\begin{tabular}{|c|c|c|c|c|c|c|c|c|c|c|c|}
\hline \multirow{2}{*}{$\begin{array}{l}\text { Sl. } \\
\text { No. }\end{array}$} & \multirow[t]{2}{*}{ Treatment } & \multirow{2}{*}{$\begin{array}{l}\text { Dosage } \\
\text { (ml or } \\
\mathrm{g} / \mathrm{l})\end{array}$} & \multicolumn{6}{|c|}{ Whitefly population* (per six leaves) } & \multirow[t]{2}{*}{ Mean } & \multirow{2}{*}{$\begin{array}{l}\text { Per cent } \\
\text { reduction over } \\
\text { control }\end{array}$} & \multirow{2}{*}{$\begin{array}{c}\text { Yield } \\
\text { (Kg/ha) }\end{array}$} \\
\hline & & & $1 \mathrm{DBS}$ & 1 DAS & 3 DAS & 5 DAS & 7 DAS & 10 DAS & & & \\
\hline 1. & Triazophos $40 \%$ EC & $2 \mathrm{ml}$ & $\begin{array}{l}21.85 \\
(4.73)\end{array}$ & $\begin{array}{c}17.66 \\
(4.26) \mathrm{ab}\end{array}$ & $\begin{array}{c}11.05 \\
(3.40) b\end{array}$ & $\begin{array}{c}5.88 \\
(2.53) \mathrm{b}\end{array}$ & $\begin{array}{c}4.90 \\
(2.32) \mathrm{a}\end{array}$ & $\begin{array}{c}5.98 \\
(2.55) \mathrm{a}\end{array}$ & 11.22 & 57.72 & 1301 \\
\hline 2. & Profenophos $50 \%$ EC & $2 \mathrm{ml}$ & $\begin{array}{l}20.92 \\
(4.63)\end{array}$ & $\begin{array}{c}18.27 \\
(4.33) \mathrm{b}\end{array}$ & $\begin{array}{c}14.55 \\
(3.88) d\end{array}$ & $\begin{array}{c}11.55 \\
(3.47) d\end{array}$ & $\begin{array}{c}10.57 \\
(3.33) \mathrm{c}\end{array}$ & $\begin{array}{c}11.65 \\
(3.49) \mathrm{cd}\end{array}$ & 14.59 & 45.02 & 1136 \\
\hline 3. & Fipronil $5 \% \mathrm{SC}$ & $1 \mathrm{ml}$ & $\begin{array}{l}22.30 \\
(4.77)\end{array}$ & $\begin{array}{c}17.02 \\
(4.19) \mathrm{ab}\end{array}$ & $\begin{array}{c}13.30 \\
(3.71) \mathrm{c}\end{array}$ & $\begin{array}{c}10.30 \\
(3.29) \mathrm{c}\end{array}$ & $\begin{array}{c}9.32 \\
(3.13) \mathrm{b}\end{array}$ & $\begin{array}{c}10.40 \\
(3.30) \mathrm{b}\end{array}$ & 13.77 & 48.11 & 1169 \\
\hline 4. & Buprofezin $25 \%$ SC & $1 \mathrm{ml}$ & $\begin{array}{l}20.70 \\
(4.60)\end{array}$ & $\begin{array}{c}17.57 \\
(4.25) \mathrm{ab}\end{array}$ & $\begin{array}{c}16.81 \\
(4.16) f\end{array}$ & $\begin{array}{c}10.85 \\
(3.37) \mathrm{cd}\end{array}$ & $\begin{array}{c}9.87 \\
(3.22) b c\end{array}$ & $\begin{array}{c}10.95 \\
(3.38) b c\end{array}$ & 14.46 & 45.51 & 1173 \\
\hline 5. & Azadirachtin $0.03 \%$ & $5 \mathrm{ml}$ & $\begin{array}{l}21.30 \\
(4.67)\end{array}$ & $\begin{array}{c}24.85 \\
(5.03) \mathrm{c}\end{array}$ & $\begin{array}{c}18.85 \\
(4.40) \mathrm{g}\end{array}$ & $\begin{array}{c}12.85 \\
(3.65) \mathrm{e}\end{array}$ & $\begin{array}{c}11.87 \\
(3.52) \mathrm{cd}\end{array}$ & $\begin{array}{c}12.95 \\
(3.67) d\end{array}$ & 17.11 & 35.53 & 1125 \\
\hline 6. & Flonic amid $50 \% \mathrm{WG}$ & $0.4 \mathrm{~g}$ & $\begin{array}{l}20.00 \\
(4.60)\end{array}$ & $\begin{array}{c}16.77 \\
(4.16) \mathrm{a}\end{array}$ & $\begin{array}{c}16.04 \\
(4.07) \mathrm{e}\end{array}$ & $\begin{array}{c}10.05 \\
(3.25) \mathrm{c}\end{array}$ & $\begin{array}{c}9.07 \\
(3.09) \mathrm{b}\end{array}$ & $\begin{array}{c}10.15 \\
(3.26) \mathrm{b}\end{array}$ & 13.80 & 48.00 & 1176 \\
\hline 7. & Diafenthiuron $50 \% \mathrm{WP}$ & $1 \mathrm{~g}$ & $\begin{array}{l}20.50 \\
(4.58)\end{array}$ & $\begin{array}{c}17.44 \\
(4.24) \mathrm{ab}\end{array}$ & $\begin{array}{c}10.13 \\
(3.26) \mathrm{a}\end{array}$ & $\begin{array}{c}5.13 \\
(2.37) \mathrm{a}\end{array}$ & $\begin{array}{c}4.15 \\
(2.16) \mathrm{a}\end{array}$ & $\begin{array}{c}5.23 \\
(2.39) \mathrm{a}\end{array}$ & 10.43 & 60.70 & 1346 \\
\hline 8. & Clothianidin $50 \% \mathrm{WG}$ & $0.1 \mathrm{~g}$ & $\begin{array}{l}21.50 \\
(4.69)\end{array}$ & $\begin{array}{c}17.59 \\
(4.25) \mathrm{ab}\end{array}$ & $\begin{array}{c}16.91 \\
(4.17) f\end{array}$ & $\begin{array}{c}10.87 \\
(3.37) \mathrm{cd}\end{array}$ & $\begin{array}{c}9.89 \\
(3.22) \mathrm{bc}\end{array}$ & $\begin{array}{c}10.97 \\
(3.39) b c\end{array}$ & 14.62 & 44.91 & 1130 \\
\hline 9. & Control & - & $\begin{array}{l}20.90 \\
(4.63)\end{array}$ & $\begin{array}{c}28.74 \\
(5.41) \mathrm{d}\end{array}$ & $\begin{array}{c}29.27 \\
(5.46) \mathrm{h}\end{array}$ & $\begin{array}{c}26.41 \\
(5.19) \mathrm{f}\end{array}$ & $\begin{array}{c}26.79 \\
(5.22) \mathrm{e}\end{array}$ & $\begin{array}{c}27.11 \\
(5.25) \mathrm{e}\end{array}$ & 26.54 & 0 & 852 \\
\hline \multicolumn{3}{|c|}{$\mathrm{SEM} \pm$} & 1.02 & 0.05 & 0.02 & 0.05 & 0.06 & 0.05 & - & - & 9.00 \\
\hline \multicolumn{3}{|c|}{ CD@5\% } & NS & 0.13 & 0.08 & 0.15 & 0.19 & 0.18 & - & - & 28.00 \\
\hline \multicolumn{3}{|c|}{$\mathrm{CV} \%$} & 8.55 & 11.21 & 10.63 & 10.51 & 13.28 & 9.72 & - & - & 10.54 \\
\hline
\end{tabular}

DBS- days before spraying, DAS-days after spraying, Figures in the parenthesis are $\sqrt{x}+0.5$ transformed values, NS= Non significant, Means followed by same letter in the column do not differ significantly by DMRT $(P=0.05)$, *Means of three replications. 


\section{References}

Heiser, C. B., 1951, The sunflower among the North American Indians. Proc. Am. Philos. Soc., 95: 432-448.

Anonymous, 2016, (http://agricoop.nic.in/ agristatistics.htm.).

Rajmohan N. Pest complex of sunflower - a bibliography. PANS. 1976; 22:546563.

Basappa H. Insect pest management in sunflower - innovative approaches. In: Subject matter workshop cum seminar on integrated pest management in oilseeds. Directorate of Oilseeds Research, Hyderabad. 1995.

Anonymous., 1997, Annual progress report of AICRP on oilseeds (Sunflower), Directorate of oilseeds Research, ICAR, Hyderabad, India, pp. 167.

Chander Rao, S., 2002, First report of tobacco streak virus infecting safflower (Carthemus tinctorius) in Maharashtra, India. Plant Disease, 87:1396.

Govindappa, M. R., Shankergoud, I., Shankarappa, K.S., Wickramaarachchi, W.A.R.T., Anjeneya Reddy, B. and Rangaswamy, K.T., 2011. Molecular detection and partial characterization Begomovirus associated with leaf curl disease of sunflower (Helianthus annuиs) in southern India. Pl. Path. J., 10: $29-35$

Katti, P., 2007, Sucking pests of sunflower with special reference to Thrips palmi Karny, its relation with necrosis virus and management. Ph.D. Thesis, Univ. Agric. Sci., Dharwad.

Vindhyashree, M., 2014, Studies on sunflower leaf curl virus (SuLCV) disease with special reference to epidemiology. M.Sc. (Agri) Thesis, Univ. Agri. Sci., Raichur.

Kharel, S., Singh P. S. and Singh, S. K., 2016, Efficacy of newer insecticides against sucking insect pests of greengram [Vigna radiata (L.) Wilczek], Int. J. of Agri, Envi and Biot., 9(6): 1081-1087.

Dhaka, S. R., 2016, Evaluation of newer insecticides against sucking pests of mungbean. Bhartiya Krishi Anushandhan Patrika, 31(3): 197-199.

Gopal Das and Tarikul Islam, 2014, Relative efficacy of some newer insecticides on the mortality of jassid and whitefly in brinjal. Int. J. Res. Biol. Sci., 4(3): 8993.

Sanjeev Kumar and Gill, C. K., 2010, Incidence of tomato leaf curl virus in relation to whitefly, Bemisia tabaci (Genn.) population in different insecticidal treatments on tomato crop. J. Insect Sci., 23(3): 327-331.

Kumar, B. A., Rao, B. N. and Sriramulu, M., 2001, Efficacy of some mixed formulations of insecticides against whitefly, Bemisia tabaci Gennadius on Brinjal. J. Res. ANGRAU. 29(2): 1721.

\section{How to cite this article:}

Praveen B. Huded, V.N. Ghante and Vikas Kulkarni. 2019. Chemical Management of Whiteflies in Sunflower. Int.J.Curr.Microbiol.App.Sci. 8(04): 2885-2890.

doi: https://doi.org/10.20546/ijcmas.2019.804.337 